\title{
Enhancing Video Streaming in 802.11 Wireless Mesh Networks using Two-Layer Mechanism Solution
}

\author{
N. H. Moleme*, M.O. Odhiambo*, A.M. Kurien ${ }^{* *}$ \\ French South African Technical Institute in Electronics \\ Tshwane University of Technology, Private Bag X680 Pretoria 0001 Republic of South Africa \\ Tel: (+27 12) 382-4191 Fax: (+27 12) 382-5294 \\ nametshego.m@gmail.com, ohangmo@unisa.ac.za, kurienam@tut.ac.za
}

\begin{abstract}
Multihop Wireless Mesh Networks have emerged as the next generation in wireless network technology. This is due to the advantages provided by the mesh network technology. WMNs provides ease of installation, cost effective deployments, high level of scalability, wide coverage area and capacity, network flexibility and self-configuration capabilities. However, despite these advantages, many research challenges still remain in WMNs. One such challenge is the support of real time applications such as video streaming. This paper proposes a two-layer mechanism for the transportation of real-time video. In this mechanism, rate adaptation is implemented in the data link layer for channel error control, link stability and reliability. In addition, the network layer routing protocol is optimized for congestion control and optimal route selection by using congestion information from the data link layer and link quality metric from the network layer. Simulation results show that the proposed mechanism improves the network performance in terms of throughput, delay and jitter in multihop wireless mesh networks when UDP is used as the transport protocol.
\end{abstract}

Index Terms-Cross-Layer, Video Streaming, Rate Adaptation, Routing Protocol.

\section{INTRODUCTION}

$\mathrm{W}$ ireless Mesh Networks have gained immense research interest from the wireless networks research community. This is due to the advantages provided by WMNs. However, there are still several research challenges that need to be addressed in the protocol layers for WMNs to support video streaming applications. In this paper, the focus is on transport layer protocol. TCP and UDP are the two prominent transport protocols used within wireless networking. However, TCP is unsuitable for real-time applications as it incorporates retransmissions and congestion control mechanisms [2], [3].UDP does not incorporate error recovery mechanisms. Thus, UDP has lower delays as compared to TCP. The absence of error recovery mechanisms makes UDP unreliable and this leads to a higher degree of packet loss probability in wireless networks. Thus, video streaming in wireless networks using UDP protocol can result in unpredictable link degradation and poor video quality. Therefore, packet error probability is maximized when more hops are encountered from source to destination. As a result, there is a need for mechanisms that optimizes throughput and improves end-to-end delays and jitter when using UDP as the transport protocol for video streaming applications in WMNs. In this paper, the data link layer and network layer are enhanced to facilitate the use of UDP for video streaming applications.

\section{RELATED WORK}

Several approaches have been proposed to improve the performance of UDP in wireless multihop networks. Bansal et al. [4] proposed a WANET routing protocol that selects optimal routes based on the link bandwidth. The proposed protocol exploits the multi-rate capability of IEEE 802.11 wireless cards to select high bandwidth routes. The rationale behind this mechanism is that minimum hop count is not sufficient as a routing metric. The inefficiencies of minimum hop count are indicated in [1], [5]. Larzon et al. [6] adopted a different approach in which they propose a modified version of the original UDP protocol termed UDP-Lite. UDP-Lite uses a partial checksum by dividing packets into sensitive and insensitive parts in the UDP header. Errors in the sensitive part results in dropped packets, while errors in the insensitive part results in packets not being dropped. This mechanism has two major drawbacks. Firstly, UDP-Lite is backward incompatible with conventional UDP in that it requires modifications to be made on the conventional UDP to interwork with it. Secondly, due to the partial checksum, application instabilities may result as certain applications may be incapable of handling erroneous packets. Boyce et al. [3] proposed a UDP protocol known as CUDP. It uses channel error information obtained from the PHY and link layers in the protocol stack to assist with error recovery at packet level. The disadvantage of this approach is that it requires information exchange from RLP layer to PPP / IP/ UDP and application layer. However, currently, information exchange from RLP to PPP/IP/UDP is not supported by the protocol stack. Thus, a redesign of the protocol stack is required. The work that is most closely related to this study is Yang [7] in which the author adopts a cross-layer design approach similar to this study. However, in contrast to [7], rate adaptation is implemented without any modifications to the IEEE 802.11 MAC layer design in the protocol stack in this study. In addition, the routing protocol in [7] uses minimum hop count metric which has been shown by [1], [5] to be inefficient. In this study, link quality metric which 
optimizes throughput, reduces packet loss and improves end-to-end delays is integrated into the routing protocol for optimal path selection.

\section{Cross-Layer Design TeChniQue}

In Wireless networks most packet errors are due to channel conditions. The simple mechanism of UDP does not guarantee delivery of packets. The UDP throughput degrades with increasing hop count in multihop networks. Combining these factors makes UDP unsuitable for video streaming in WMNs. The proposed scheme aims at meliorating the performance of UDP in WMN video streaming applications by improving throughput, packet loss and latency.
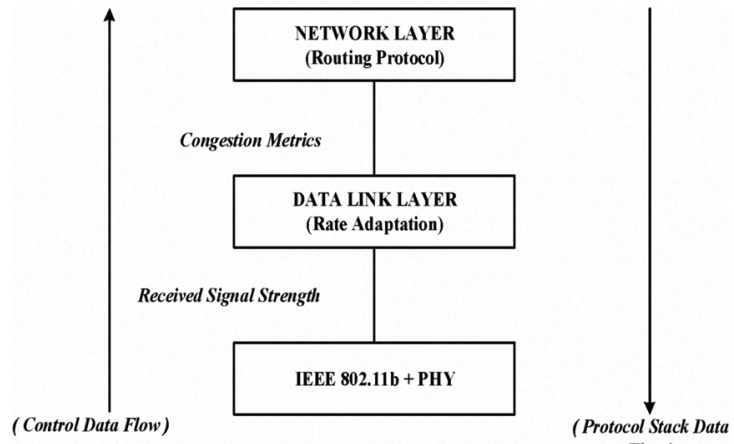

Flow)

Figure 1: Implementation of the Cross-Layer Design in the Protocol Stack

This mechanism comprises of rate adaptation and routing optimization, which is implemented in the protocol stack as shown on Figure 1. The rate adaptation scheme adapts the transmission rate of the sender in the MAC layer based on the channel estimation information from the PHY layer and the routing protocol utilizes congestion metrics from MAC layer and network layer to select least congested routes to enhance the performance of the $\mathrm{WMN}$ for video streaming applications.

\section{A. Rate Adaptation Scheme}

The rate adaptation technique used in this study is based on a SNR automatic rate control technique as discussed in [8]. The principle of this rate adaptation technique is to dynamically change the data transmission rate based on the channel quality estimation SNR which is directly related to the BER in the link and ultimately the FER. Consequently, the SNR is linked to the packet delay, jitter and throughput. It holds the potential of providing rich feedback for automatic rate control. Knowledge of the current SNR and the throughput vs. the SNR curves for each rate setting solves the rate-selection problem. The automatic rate controller simply selects the optimal rate from the throughput vs. the SNR curves for a specific 802.11 technology. An example of these curves which indicates the attainable throughput for different transmission rates based on the channel conditions is shown in Figure 2. It is shown in Figure 2 that the throughput is dependent on the modulation schemes given the SNR. For instance, it is shown that to maximize the throughput, 64 QAM $1 / 2$, must be used at $27.5 \mathrm{~dB}$ to $32.5 \mathrm{~dB}$, while 64 QAM $3 / 4$ must be used at $32.5 \mathrm{~dB}$ to $45 \mathrm{~dB}$. However, if 16 QAM $1 / 2$ is used between $32.5 \mathrm{~dB}$ to $45 \mathrm{~dB}$, the throughput will be reduced by a significant margin of $1.1 \mathrm{Mbps}$. The SNR-based automatic rate control has not been widely applied in practice despite its advantages of being a fast response, rich feedback and easily implemented mechanism [8]. This is mainly due to the following reasons: Firstly, in reality, for certain link conditions, the relation between the optimal rate and SNR is highly variable. Secondly, it is not crucial to obtain a reliable estimate of the SNR of a link because many radio interfaces provide only an uncalibrated SSI. Thirdly, the rate controller, which is at the sending side, needs the SNR observed at the receiving side.

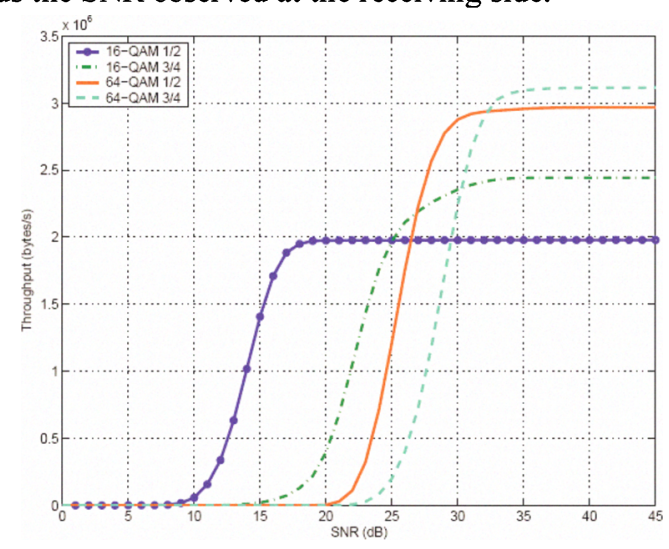

Figure 2: Throughput vs. SNR for some 802.11a modulation schemes

To overcome the above mentioned issues, a proper selection of a channel model which has been practically proven to be accurate in estimating the channel conditions in the IEEE 802.11 needs to be done.

\section{Channel Model}

It has been shown in practice through practical experiments that the log-distance model can be used to estimate the IEEE 802.11wireless channels both indoors and outdoors with reasonable accuracy [9].The experiments produced a standard deviation of $7.36 \mathrm{~dB}$ indoors and 3.1 $\mathrm{dB}$ outdoors. The log-distance model is expressed mathematically as:

$$
\overline{P L(d B)}=\overline{P L\left(d_{0}\right)}+10 \cdot n \cdot \log \left(\frac{d}{d_{0}}\right)+X_{\sigma}
$$

Where $n$ is the path loss exponent for different propagation environments, $d$ is the distance between the transmitter and the receiver, $d_{0}$ is the close-in reference distance.

\section{Throughput Model}

According to [8], [10] to model throughput, the time taken to transmit a packet and the packet length needs to be known as indicated by equ 2 . The packet length refers to the MSDU length.

$$
\begin{aligned}
& T\left(L_{M S D U}, T_{\text {frame }}\right)=\left(\frac{L_{M S D U} \cdot 8}{T_{\text {frame }}}\right) M b p s \\
& \left.T_{\text {frame }}=T_{S}(0)+\sum_{i=1}^{+\infty} 1-p_{\text {framerrot }}\right) \cdot\left(P_{\text {framerrot }}\right)^{i}\left[\sum_{j=0}^{i-1} T_{f}(j)+T_{S}(i)\right] \mu,
\end{aligned}
$$

where $T_{\text {frame }}$ is the average transmission time for a single frame including retransmission. To model $T_{\text {frame }}, \mathrm{MAC}$ 
protocol DCF mechanism without RTS and CTS process as in equ 3. In IEEE 802.11 a packet is encapsulated in a MSDU from the network layer to the MAC layer.

\section{Rate Controller}

For the automatic rate controller, a simple threshold based technique was used. In which the transmission rate is chosen based on the channel quality estimation and the maximum attainable throughput under the estimated channel conditions. The RSS is used as the Channel Status Indicator. The transmission power of each MSDU is known, therefore, the receive power is estimated using the channel model indicated in equ 1. The RSS is then mapped to a transmission data rate based on the estimated maximum attainable throughput modeled in equ 2.

\section{B. Routing optimizations}

To optimize the routing in the network layer for multihop communication, OLSR is enhanced to utilize congestion information from MAC layer and link quality metric in the network layer.

\section{Link Quality Metric Based Modifications}

The link quality metric routing modifications are based on information found in [5].Most existing wireless Ad Hoc routing protocols commonly use minimum hop count as a routing metric[5].Firstly, it assumes that links are binary meaning they either exist or don't exist. Secondly, it maximizes the distance traveled by each hop, which is likely to minimize signal strength and maximize the loss ratio. Thirdly, even if the best route is a minimum hopcount, in a dense network there may be many routes of the same minimum length, with widely varying qualities; thus, the arbitrary choice made by most minimum hop-count metrics is not likely to select the best routes. It is shown [5] through practical experiments that using minimum hop count as a routing metric often leads to less capacity than the existing best paths due to link asymmetry, link quality variations and an almost non existent correlation between link signal strength and delivery rate.ETX metric of a link is the predicted number of data transmissions required to send a packet over that link and is calculated as:

$$
E T X=\frac{1}{d_{f} \mathrm{X} d_{r}}
$$

where $d_{f}$ is the forward delivery ratio and $d_{r}$ is the reverse delivery ratio. The delivery ratio from the sender at any time $t$ is:

$$
r(t)=\frac{\operatorname{Count}(t-w, t)}{w / \tau}
$$

where count $(t-w, t)$ is the number of link probes received during window $w$ and $w / \tau$ the number of probes that should have been received in the same period. The route that minimizes ETX is selected as the best path for routing. To incorporate link quality metric into the routing protocol and implement it, the delivery ratios are measured using modified HELLO messages that are sent every $\tau=1 \mathrm{~s}$ in the simulations. The window period $(w)$ is set at $10 \mathrm{~s}$ in the simulations. Thus, $w / \tau$ is 10 in the simulations. The ETX value is set to 0 if there are no HELLO messages received during the set $w$ period and the link is not considered for routing.

\section{Congestion Optimization}

The congestion information at each node is obtained based on the method presented in [11]. The average MAC layer utilization level indicates the degree to which the wireless medium around that node is busy or idle. The value is averaged within a period (10s) to obtain an indication of the use of the wireless medium around the node. The other metric that is used is the instantaneous transmission queue length at the network layer. It is based on the notion that in certain cases, a node may not be experiencing much MAC layer congestion, but instead may have many packets backlogged and if that node is chosen to forward other packets, it may result in an increased packet latency or even dropped packets due to a limit that is imposed on the queue length. To enable the usage of congestion information in the network layer routing protocol, ETX calculation process is modified. From equ 4, the ETX of a lossless link is 1 . If the link is degraded the ETX value will be greater than 1 . When a neighbour node receives HELLO messages, it calculates the ETX value. The node will then check its congestion metrics and compares the values against preset thresholds. In the simulations, MAC layer utilization was set at $15 \%$ and Interface Queue Length 10 to indicate a high level of congestion. If the congestion metrics are higher than the indicated thresholds, ETX is increased; otherwise ETX remains as it was before congestion measurement. The rationale behind this method is that in multihop networks the path with the minimum ETX value is selected as the best path. Thus, increasing the ETX value will enable the routing algorithm to select the least congested routes as ETX will be minimized as compared to congested routes.

\section{Performance Evaluation}

This section presents the simulation tests undertaken to evaluate the performance of the proposed mechanism. The rationale behind the proposed approach is that the performance of the UDP can be significantly improved for video streaming applications when the cross-layer method is incorporated into WMNs.

\section{A. Rate Adaptation Implementation}

In throughput evaluations, the BER performance for different transmission rates is established using methods found in [10] which give the bit error probability when transmitting an IEEE 802.11 frame. The throughput performance for a fixed maximum MSDU length of 1500 bytes is shown in Figure 3.It observed that an $8 \mathrm{Mbps}$ throughput is achieved for $11 \mathrm{Mbps}$ transmission rate yielding a difference of $3 \mathrm{Mbps}$. However, this reduction in throughput is expected as it is commonly known that in IEEE 802.11 the actual expected throughput and the transmission rate cannot match due to MAC and PHY layer overheads such as the MAC header, PLCP preamble and PLCP header. RSS was used instead of SNR as it is a more 
practical parameter that is available in NS-2 simulations. The rate controller thresholds are extracted from Figure 3 and used in NS-2 simulations to implement and evaluate the effect of rate adaptation on video streaming applications.

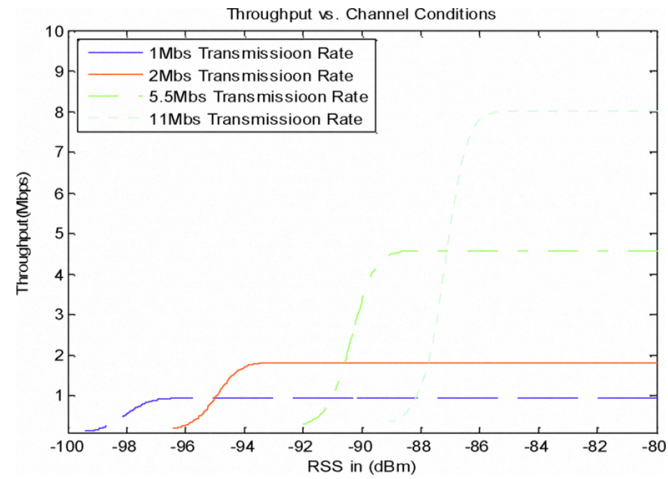

Figure 3: Throughput Performance for IEEE 802.11b Transmission Rates

\section{B. Simulations and Results}

The optimized routing protocol is benchmarked against AODV and DSR routing protocols. The first scenario, the WMN is characterized by 4 nodes and video traffic connection. In the second scenario, 11 more nodes are added to the network to establish a large and dense WMN.

\section{Scenario 1}

The results achieved from the first scenario are depicted in Figure 4 to Figure 6. Figure 4 illustrates the throughput performance of the AODV, DSR and OLSR routing protocols. It is shown in Figure 4 that the AODV and DSR perform better in comparison to the OLSR in this scenario. Taking 100 s as the reference point, it is shown that both the AODV and DSR achieve a throughput of $185 \mathrm{kbps}$ while the OLSR manages $178 \mathrm{kbps}$. Thus, the two reactive routing protocols outperform the proactive routing protocol by a margin of $3.8 \%$ in terms of throughput.

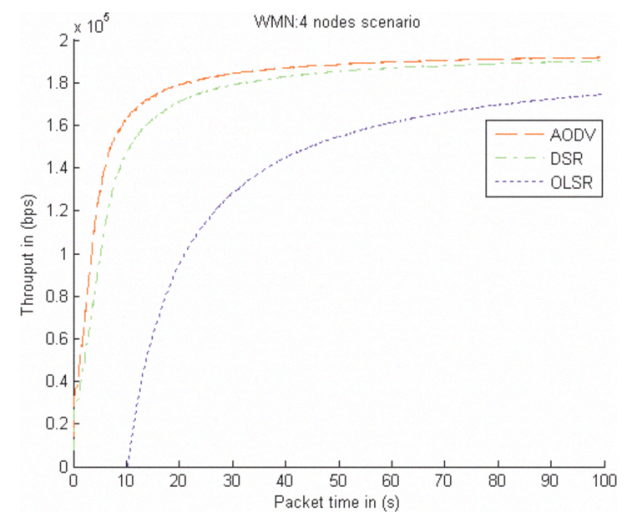

Figure 4: Throughput performance for reactive routing protocols vs. link state proactive routing protocol

In Figure 5, the delay performance AODV and DSR against OLSR is depicted. It is shown in Figure 5 that the DSR performs better than both the AODV and OLSR. Comparing the delay of the three protocols at a reference point of $25 \mathrm{~s}$, it is shown that DSR outperforms AODV by a margin of $45 \%$ and the OLSR by $54 \%$. It is also shown in Figure 5 that the as time increases, the delay margin between the OLSR and AODV is reduced.

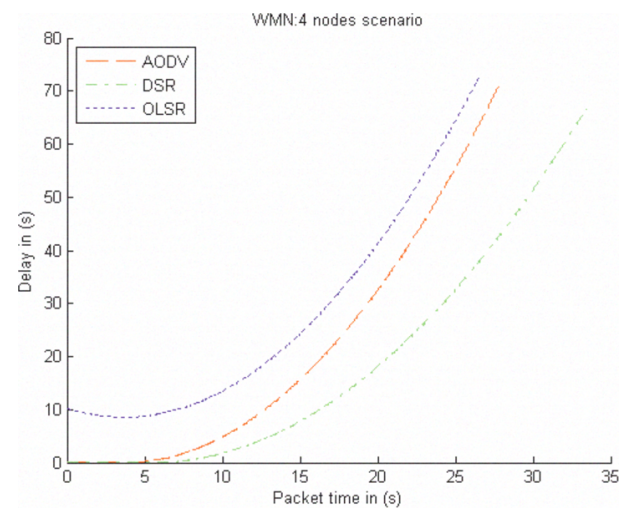

Figure 5: Delay performance for reactive routing protocols vs. link state proactive routing protocol

In Figure 6, the delay jitter comparison of the two reactive routing protocols against the proactive link state routing protocol is shown. The results shown in Figure 6 indicate that the delay jitter performance is almost similar for all three protocols averaging to $0.02 \mathrm{~s}$ within the time interval.
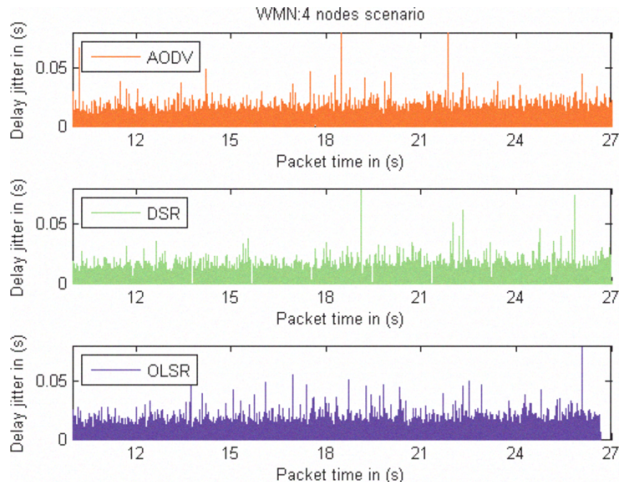

Figure 6: Delay jitter performance for reactive routing protocols vs. link state proactive routing protocol

\section{2. $\quad$ Scenario 2}

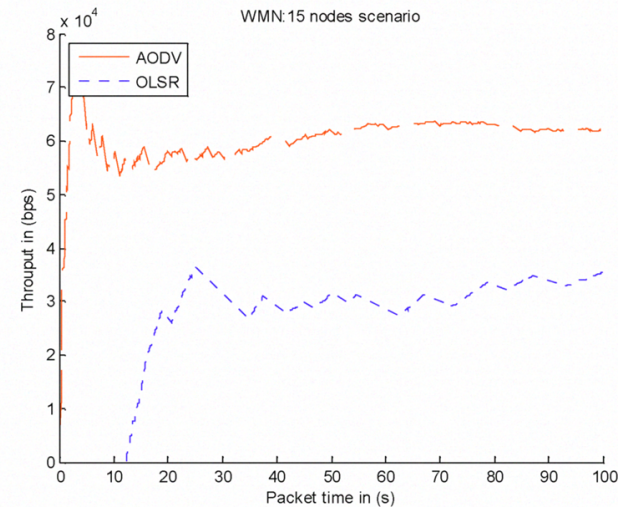

Figure 7: Throughput performance for on-demand reactive routing protocol vs. link state proactive routing protocol 
In Figure 7, the throughput performance comparison between the AODV and OLSR of scenario 2 is shown. The throughput curves shown in Figure 7 indicate that AODV still achieved a better throughput compared to the OLSR in scenario 1. At $100 \mathrm{~s}, \mathrm{AODV}$ achieved a throughput of 60 kbps compared to the $35 \mathrm{kbps}$ achieved by OLSR. Thus, AODV was found to outperform OLSR by a margin of $41 \%$.In Figure 8 , the delay performance comparison between the AODV and OLSR is shown. Comparing the two protocols from $0 \mathrm{~s}$ to $15 \mathrm{~s}$, it is shown that OLSR performance improves as time increases in scenario 2. At a reference point of $5 \mathrm{~s}$, the OLSR delay is $12 \mathrm{~s}$ while the AODV delay is 8s. This shows that AODV outperforms OLSR by a margin of $33 \%$. However, at a reference point of $15 \mathrm{~s}$, it is shown that OLSR outperforms AODV by a margin of $56 \%$.

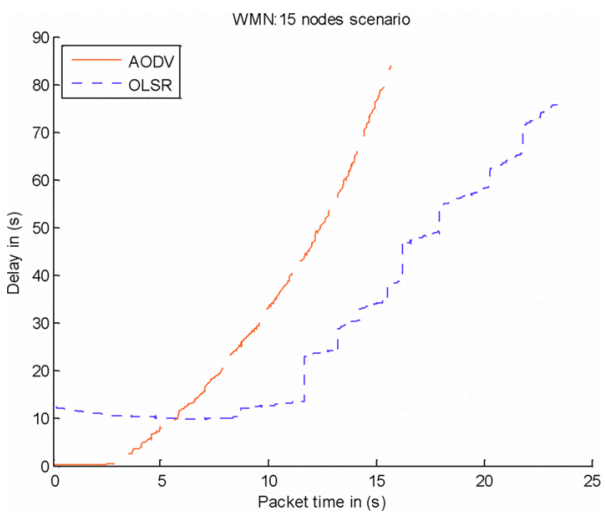

Figure 8: Delay performance for on-demand reactive routing protocol vs. linkstate proactive routing protocol

In Figure 9, the delay jitter performance of the ondemand reactive AODV and link state proactive OLSR routing protocols in scenario 2 is shown. OLSR achieved an average jitter of $0.03 \mathrm{~s}$ while AODV managed an average jitter of $0.04 \mathrm{~s}$. Thus, OLSR outperformed AODV by a margin of $25 \%$ in terms of jitter.
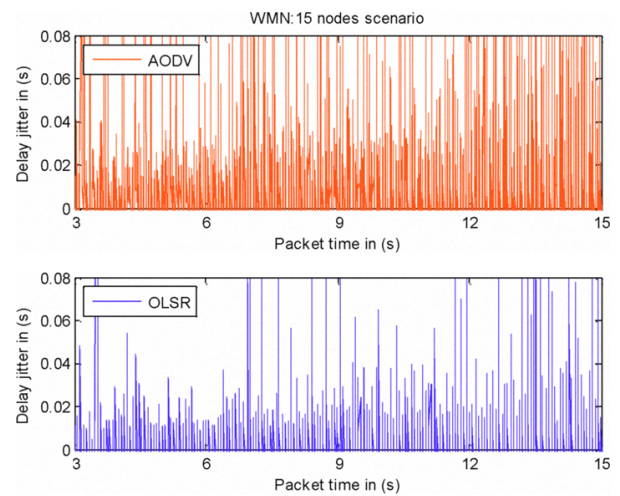

Figure 9: Delay jitter performance for on-demand reactive routing protocol vs. link state proactive routing protocol

The analysis of the results achieved from the presented scenarios provides certain significant observations. The first observation is that UDP throughput degrades with an increase in the number of hops. For instance, in scenario 1, AODV achieves a throughput of $185 \mathrm{kbps}$ when compared to the $60 \mathrm{kbps}$ achieved in scenario 2 , resulting in a $67 \%$ throughput degradation. The second observation is that as the network is made larger and denser, the proactive OLSR outperforms the reactive AODV in terms of delay and jitter, as indicated by Figure 8 and Figure 9 respectively. Thus, the conclusion drawn from this observation is that the OLSR is well-suited for real time applications which are delay sensitive.

\section{Congestion Based Routing Optimization Evaluations}

The OLSR ETX is modified for congestion optimization and termed OLSR CON. To evaluate the performance of OLSR CON which is optimized for congestion, three video source-destination connections are established within the 15 node multi-hop network. Figure 10 illustrates the relative throughput performance of the routing protocols under the specified traffic load.

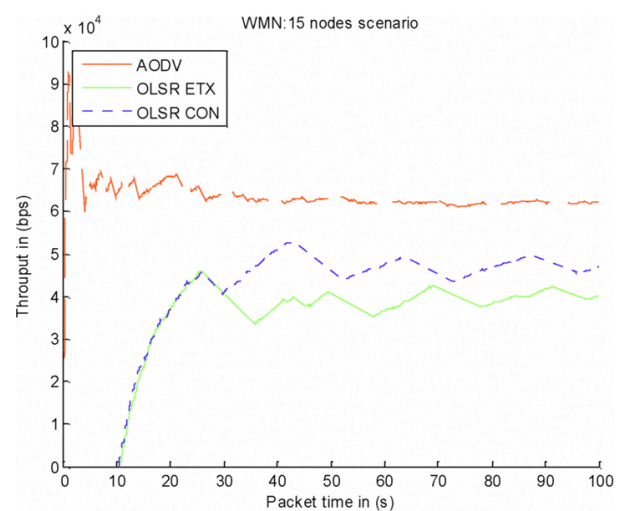

Figure 10: Throughput performance for on-demand reactive routing protocol vs. linkstate proactive routing protocol with congestion optimization

It is shown in Figure 10 that the congestion based modifications result in significant improvements in terms of throughput. Taking a reference point of $100 \mathrm{~s}$, AODV outperforms OLSR ETX by a margin of $33 \%$ and OLSR CON by $25 \%$. This indicates that OLSR CON improved throughput by a significant margin of $8 \%$. An important observation is that AODV still outperforms OLSR CON in terms of throughput.

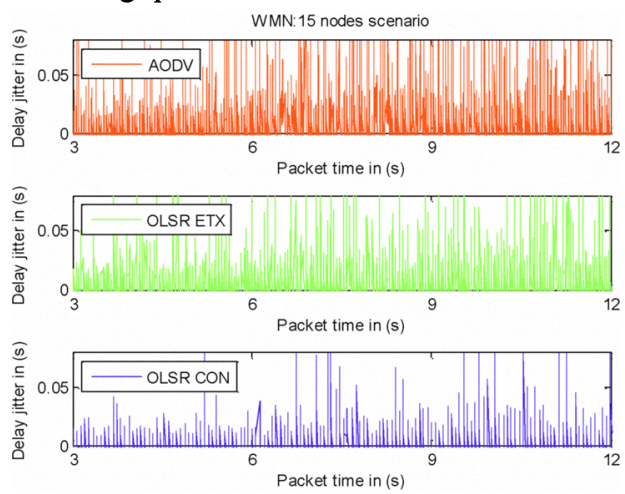

Figure 11: Delay jitter performance for on-demand reactive routing protocol vs. link state proactive routing protocol with congestion optimization 
In Figures 11, the jitter performances of the AODV, OLSR ETX and OLSR CON are shown. The results indicate that the OLSR CON has a better performance compared to both the AODV and OLSR ETX in terms of jitter. It is shown in Figure 11 that the OLSR CON achieved an average jitter of $0.025 \mathrm{~s}$ compared to the $0.035 \mathrm{~s}$ and $0.045 \mathrm{~s}$ achieved by the OLSR ETX and AODV respectively. Thus, the OLSR CON outperforms the OLSR ETX by a margin of $28 \%$ and the AODV by $44 \%$. The results shown in Figure 10 to Figure 11 indicate that the congestion optimization results in significant routing optimizations such as the OLSR CON achieved significant improvements in terms of throughput, delay and jitter.

\section{Rate Adaptation Evaluations}

The required successful reception and decoding of a packet is dependent on the transmission rate, RSS (RxThresh) and SINR (CPThresh). Rate adaptation evaluation is based on the OLSR CON routing protocol which is optimized for both link quality and congestion. The results shown in Figure 13 indicate that $1 \mathrm{Mbps}$ achieves the highest throughput of $225 \mathrm{kbps}$ compared to other transmission rates at $100 \mathrm{~s}$. The rate adaptation scheme implemented in this study selects $1 \mathrm{Mbps}$ as the optimum transmission rate as this rate achieves the highest throughput. When comparing the results indicated in Figure 13 to the results in Figure 10. Taking 100s as the reference point, the OLSR CON achieves a throughput of $50 \mathrm{kbps}$ at $11 \mathrm{Mbps}$ and $225 \mathrm{kbps}$ at $1 \mathrm{Mbps}$. This indicates that the rate adaptation has improved throughput by $78 \%$. In comparison to the AODV, the OLSR CON (1Mbps transmission rate) achieves a $73 \%$ improvement in terms of throughput. This demonstrates that the proposed rate adaptation method improves the overall throughput performance in multi-hop wireless networks. It should be noted that lower rates occupy more channel time than higher rates, as mentioned before. The end-to-end delay is significantly reduced in the OLSR CON, which selects routes with a high quality and less congestion.

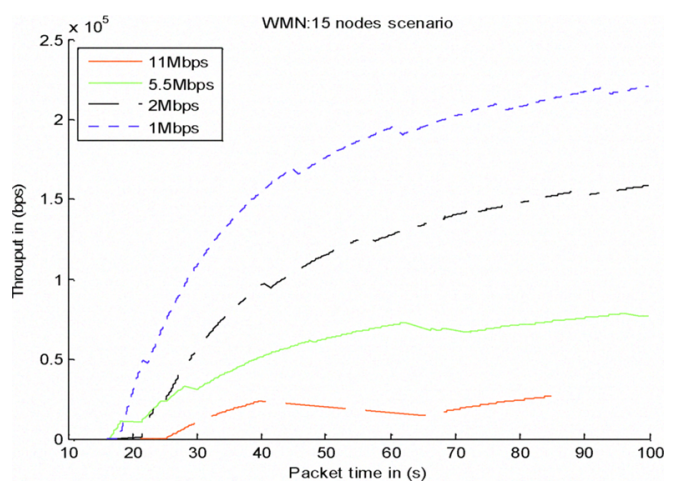

Figure 12: Throughput performance for different IEEE 802.11b transmission rate in a multihop wireless network

\section{Conclusion}

The outcomes of the paper present substantial evidence that the proposed mechanism provide a significant framework for the enabling of WMNs to support video streaming including other real time applications. The undertaken simulation test results show that two-layer mechanism solution based on rate adaptation at the MAC layer with the incorporation of the link quality metric and congestion control mechanism in the network layer routing protocol optimizes the overall network performance.

\section{ACKNOWLEDGEMENTS}

The authors would like to thank the Telkom Centre of Excellence Program and F'SATIE at Tshwane University of Technology for making this research possible.

\section{REFERENCES}

[1] V.Chavoutier, D.Maniezo, C.E.Palazzi, M.Gerla, "Multimedia over Wireless Mesh Networks: Results from a Real Testbed Evaluation", the sixth Annual Ad hoc Networking workshop, Corfu, Greece, June 12-15, 2007.

[2] L. Larzon, M. Degermark, and S. Pink, "Efficient Use of Wireless Bandwidth for Multimedia Applications", MoMuc '99, San Diego, November 1999, pp. 187-193.

[3] H. Zheng and J. Boyce, "An Improved UDP Protocol for Video Transmission Over Internet-to-Wireless Networks", IEEETrans. On Multimedia, Vol. 3, No. 3, pp. 356-365, September 2001

[4] S. Bansal, R. Shorey, and A. Kherani, "Performance of TCP and UDP protocols in multi-hop multi-rate wireless networks", in: IEEE WCNC, Atlanta, USA, March 2004

[5] D. S. J. De Couto, D. Aguayo, J. Bicket, and R. Morris, "A high throughput path metric for multi-hop wireless routing," in Proc. ACM Int. Conf. Mobile Comput. Netw, 2003, pp. 134-146.

[6] Larzon L, Degermark. M, and Pink.S, "UDP Lite for Real Time Multimedia Applications," HP Labs, Bristol, United Kingdom, Tech Rep. HPL-IRI-1999-001, Apr. 1999.

[7] Ning Yang, "Congestion-Aware Cross-Layer Design for Wireless Ad Hoc Networks", Ph.D Thesis, University of South Florida, July 2004.

[8] Ivaylo Haratcherev, "Application-oriented Link Adaptation for IEEE 802.11", Ph.D Thesis, Delft University of Technology, March 2006, ISBN10: 90-9020513-6, ISBN-13: 978-90-9020513-7

[9] L.N. Gumbi, D. Chatelain., M. Odhiambo, "Power-Efficient Algorithm for IEEE 802.11b Multi-Hop Networks" Proc. of Southern African Telecommunication Networks \& Applications Conference (SATNAC'06), Spier Wine Estate, South Western Cape, South Africa, September 2006

[10]Liljana Gravrilovska, Vladimir Atanasovski, "Influence of Packet Length on IEEE 802.11b Throughput Performance in Noisy Channels", Proceedings of $1^{\text {st }}$ International MAGNET Workshop D6.2.5, Shangai, China, 11-12 November 2004, ISBN 87-91696-36-4.

[11] Y.-C. Hu and D. B. Johnson, "Exploiting Congestion Information in Network and Higher Layer Protocols in Multihop Wireless Ad Hoc Networks," In the 24th International conference on Distributed computing Systems (ICDCS 2004), pp.301-310, IEEE, Japan, March 2004

N.H. Moleme obtained her B.Tech Degree from Tshwane University of Technology in 2006. She is currently working towards a double degree, (M.Sc with F'SATIE, and M.Tech with Tshwane University of Technology). Her research interests include rate adaptation algorithms, network protocols, and multimedia applications in wireless networks. 